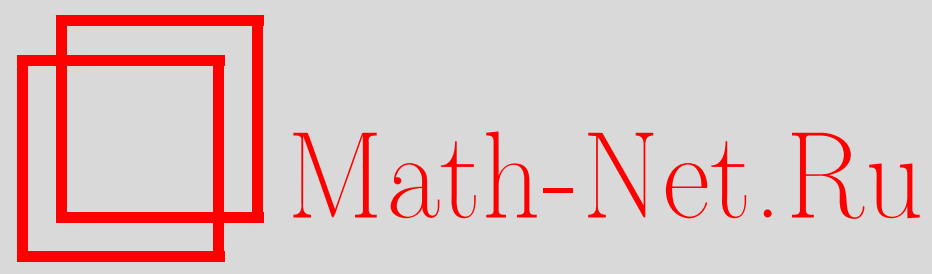

Х. Д. Икрамов, Об одном замечательном свойстве матрицы Марка Каца, Матем. заметки, 2002, том 72, выпуск 3, 356362

DOI: https://doi.org/10.4213/mzm427

Использование Общероссийского математического портала Math-Net.Ru подразумевает, что вы прочитали и согласны с пользовательским соглашением http://www . mathnet.ru/rus/agreement

Параметры загрузки:

IP: 35.173 .137 .237

26 апреля 2023 г., 17:09:14

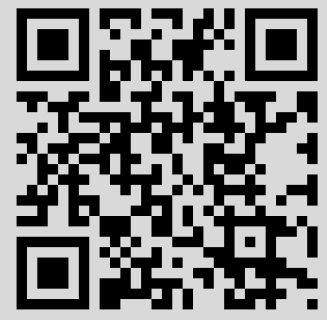




\section{ОБ ОДНОМ ЗАМЕЧАТЕЛЬНОМ СВОЙСТВЕ МАТРИЦЫ МАРКА КАЦА}

\section{Х. Д. Икрамов}

Треугольная подматрица, выделяемая специальным образом из матрицы Марка Каца, обладает замечательным спектральньм свойством: при перестановке ее столбцов в обратном порядке половина собственных значений не меняется, а остальные умножаются на -1 . Этот факт, установленный автором ранее, не имел до сих пор объяснения. Такое объяснение дается в настоящей статье.

Библиограффия: 4 названия.

1. Матрицей Марка Каца порядка $n$ назьвается (см. [1]) трехдиагональная матрица

$$
S_{n}=\left[\begin{array}{cccccc}
0 & 1 & & & & \\
n-1 & 0 & 2 & & & \\
& n-2 & 0 & 3 & & \\
& & \ddots & \ddots & \ddots & \\
& & & 2 & 0 & n-1 \\
& & & & 1 & 0
\end{array}\right] \text {. }
$$

Число $n$ в дальнейшем считается четньм: $n=2 m$.

Определим $n \times n$ матрицу-перестановку $U=\left(u_{i j}\right)$ следующим образом:

$$
u_{i j}=\delta_{i, k(j)}, \quad \text { где } k(j)= \begin{cases}2 j-1, & 1 \leqslant j \leqslant m, \\ 2(j-m), & m+1 \leqslant j \leqslant n .\end{cases}
$$

Здесь $\delta_{p q}-$ символ Кронекера. Подобие $S_{n} \rightarrow U^{T} S_{n} U$ преобразует $S_{n}$ в матрицу

$$
\left[\begin{array}{cc}
0 & K_{m} \\
L_{m} & 0
\end{array}\right]
$$

где

$$
K_{m}=\left[\begin{array}{ccccc}
1 & & & & \\
2 m-2 & 3 & & & \\
& 2 m-4 & 5 & & \\
& & \ddots & \ddots & \\
& & & 2 & 2 m-1
\end{array}\right]
$$

Именно матрица $K_{m}$ (а не сама матрица Марка Каца) является предметом исследования в данной статье. Как было показано в [2], эта матрища обладает следующим 
замечательным свойством: при изменении порядка ее столбцов на обратньй половина собственных значений не меняется, а остальные умножаются на -1 . Сопоставим данный факт с тем, что, как правило, воздействие перестановки (только) столбцов матрицы (или только ее строк) на собственные значения предсказать невозможно.

Эффект предсказуемого изменения собственных значений матрищы $K_{m}$ был зарегистрирован и доказан в [2], но причина, почему собственные значения изменяются именно таким образом, не была там объяснена. Это объяснение дается в настоящей статье.

2. Положим

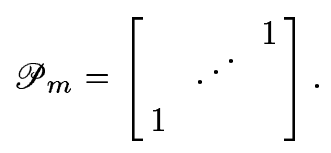

Собственньми значениями матрищы $K_{m}$ являются, очевидно, числа $1,3, \ldots, 2 m-1$. Сформулируем точный результат, касающийся собственных значений матрицы

$$
N_{m}=K_{m} \mathscr{P}_{m}
$$

Теорема 1. Спектр матрииы $N_{m}$ составляют числа

$$
2 m-1,-(2 m-3), 2 m-5,-(2 m-7), \ldots,(-1)^{m-2} 3,(-1)^{m-1} \text {. }
$$

Если собственные значения матрицы $K_{m}$ расположить в виде вектора $\lambda_{m}$ с возрастающими компонентами, то спектр матрищы $N_{m}$ можно характеризовать вектором $\mu_{m}=D_{m} \lambda_{m}$, где

$$
D_{m}=\operatorname{diag}\left((-1)^{m-1}, \ldots,-1,1,-1,1\right) .
$$

Сформулируем вкратце наше объяснение теоремы 1 (его обоснованию посвящена остальная часть статьи). Как и любая квадратная матрища, $K_{m}$ подобна своей транспонированной матрице. Для наших целей достаточно говорить о подобии над $\mathbb{R}$. Поскольку все собственные значения матрицы $K_{m}$ различны, всякая матрица $Q$, трансформирующая $K_{m}$ подобием в $K_{m}^{T}$, симметрична (см. [3], [4, с. 65]). В $n$-мерном многообразии таких матриц $Q$ имеется единственная (с точностью до умножения на ненулевую константу) матрица $Q_{0}$, обладающая дополнительными симметриями, а именно: нечетные, считая от конца, столбцы матрищы $Q_{0}$ симметричны, а четные, также считая от конща, кососимметричны. Напомним, что вектор $x \in \mathbb{R}^{m}$ называется симметричным, если $x=\mathscr{P}_{m} x$, и кососимметричным, если $x=-\mathscr{P}_{m} x$. Ясно, что симметричные (кососимметричные) векторы суть собственные векторы матрицы $\mathscr{P}_{m}$, отвечающие ее собственному значению $1(-1)$.

Указанные вьше свойства симметрии матрицы $Q_{0}$ обеспечивают справедливость матричного равенства

$$
\mathscr{P}_{m} Q_{0}=Q_{0} D_{m}
$$

Из него вьводим

$$
\begin{aligned}
N_{m} & =K_{m} \mathscr{P}_{m}=K_{m}\left(Q_{0} D_{m} Q_{0}^{-1}\right)=\left(K_{m} Q_{0}\right) D_{m} Q_{0}^{-1} \\
& =\left(Q_{0} K_{m}^{T}\right) D_{m} Q_{0}^{-1}=Q_{0}\left(K_{m}^{T} D_{m}\right) Q_{0}^{-1}
\end{aligned}
$$

Таким образом, $N_{m}$ подобна матрице $K_{m}^{T} D_{m}$. Последняя имеет спектр, указанный в $(2)$. 
3. Все матрищы $Q$ такие, что

$$
Q^{-1} K_{m} Q=K_{m}^{T}
$$

могут быть получены следующим образом. Пусть $P$ - (какая-либо) невырожденная матрица, составленная по столбцам из собственных векторов матрицы $K_{m}$. Тогда

$$
Q=P D P^{T}
$$

для некоторой невырожденной диагональной матрицы $D$.

Собственные векторы треугольной матрищы $K_{m}$ легко могут быть вычислены.

Теорема 2. Нижнетреугольная $(m \times m)$-матрица $P$ с әлементами

$$
p_{i j}=(-1)^{i-j}\left(\begin{array}{c}
m-j \\
i-j
\end{array}\right), \quad i \geqslant j
$$

есть матрица собственных векторов для матрицы $K_{m}$.

ДокАЗАТЕЛьСтво. Наряду с (1) рассмотрим матрицу

$$
K_{m-1}=\left[\begin{array}{ccccc}
1 & & & & \\
2 m-4 & 3 & & & \\
& 2 m-6 & 5 & & \\
& & \ddots & \ddots & \\
& & & 2 & 2 m-3
\end{array}\right] \text {. }
$$

Очевидно, что для собственного вектора матрицы $K_{m}$, ассоциированного с собственньм значением $2 k-1$, где $k>1$, первые $k-1$ компонент равны нулю. Остальные компоненты определяются из однородной системы линейных уравнений размера $(m-k) \times(m-k+1)$ с матрицей коэффициентов

$$
\left[\begin{array}{ccccc}
2(m-k) & 2 & & & \\
& 2(m-k-1) & 4 & & \\
& & \ddots & \ddots & \\
& & & 2 & 2(m-k)
\end{array}\right] .
$$

Точно такая же система определяет ненулевые компоненты собственного вектора матрицы $K_{m-1}$, отвечающего собственному значению $2 k-3$. Это означает, что матрица $P_{m-1}$ собственных векторов для $K_{m-1}$ вложена в $P$ как нижняя угловая подматрица. Следовательно, теорема 2 будет доказана, если проверить, что вектор

$$
w=\left(\left(\begin{array}{c}
m-1 \\
0
\end{array}\right),-\left(\begin{array}{c}
m-1 \\
1
\end{array}\right), \ldots,(-1)^{m-1}\left(\begin{array}{l}
m-1 \\
m-1
\end{array}\right)\right)^{T}
$$

есть собственный вектор матрицы $K_{m}$, соответствующий собственному значению 1 , т.е. что

$$
K_{m} w=w
$$


Для вектора в левой части $i$-я компонента $(i>1)$ равна

$$
2(m-i+1)(-1)^{i-2}\left(\begin{array}{c}
m-1 \\
i-2
\end{array}\right)+(2 i-1)(-1)^{i-1}\left(\begin{array}{c}
m-1 \\
i-1
\end{array}\right)
$$

Используя известное соотношение

$$
\left(\begin{array}{c}
m-1 \\
i-2
\end{array}\right)=\frac{i-1}{m-i+1}\left(\begin{array}{c}
m-1 \\
i-1
\end{array}\right)
$$

приводим (7) к виду

$$
(-1)^{i-1}\left(\begin{array}{c}
m-1 \\
i-1
\end{array}\right)
$$

что является $i$-й компонентой вектора $w$. Первые компоненты обоих векторов в (6) равны 1.

Отметим, что в зависимости от четности числа $m$ вектор (5) симметричен либо кососимметричен. Согласно (3) первый столбец матрищы $Q$ отличается от $w$ лишь скалярным множителем, а потому имеет тот же тип симметрии, какова бы ни была матpuи $D$. В следующих пунктах будет показано, что $D$ можно выбрать так, чтобы и остальные столбцы в $Q$ обладали симметрией либо косой симметрией.

4. Начнем с разбора случаев $m=2$ и $m=3$, где возможность найти нужную матрицу

$$
D=\operatorname{diag}\left(d_{1}, \ldots, d_{m}\right)
$$

почти очевидна.

Для $m=2$ имеем

$$
P=\left[\begin{array}{cc}
1 & 0 \\
-1 & 1
\end{array}\right] \quad \text { и } \quad Q=\left[\begin{array}{cc}
d_{1} & -d_{1} \\
-d_{1} & d_{1}+d_{2}
\end{array}\right]
$$

Второй столбец матрицы $Q$ будет симметричен при $d_{1}+d_{2}=-d_{1}$, т.е. если

$$
d_{2}=-2 d_{1}
$$

Получаемая при этом матрища $Q$ с точностью до скалярного множителя равна

$$
\left[\begin{array}{cc}
1 & -1 \\
-1 & -1
\end{array}\right]
$$

Если $m=3$, то

$$
P=\left[\begin{array}{ccc}
1 & 0 & 0 \\
-2 & 1 & 0 \\
1 & -1 & 1
\end{array}\right] \quad \text { и } \quad Q=\left[\begin{array}{ccc}
d_{1} & -2 d_{1} & d_{1} \\
-2 d_{1} & 4 d_{1}+d_{2} & -2 d_{1}-d_{2} \\
d_{1} & -2 d_{1}-d_{2} & d_{1}+d_{2}+d_{3}
\end{array}\right]
$$

Условие

$$
d_{2}=-4 d_{1}
$$


необходимо для того, чтобы второй столбец в $Q$ был кососимметричен: его средняя компонента должна быть нулевой. Условие (9) и достаточно, поскольку в этом случае третья компонента $-2 d_{1}-d_{2}=2 d_{1}$ противоположна первой.

Для симметрии третьего столбца матрицы $Q$ теперь нужно лишш, чтобы

$$
d_{1}=d_{1}+d_{2}+d_{3}
$$

т.е. чтобы

$$
d_{3}=4 d_{1} .
$$

Опуская в соответствующей матрице $Q$ множитель $d_{1}$, получаем

$$
Q=\left[\begin{array}{ccc}
1 & -2 & 1 \\
-2 & 0 & 2 \\
1 & 2 & 1
\end{array}\right]
$$

Более содержателен случай $m=4$. Здесь

$$
P=\left[\begin{array}{cccc}
1 & 0 & 0 & 0 \\
-3 & 1 & 0 & 0 \\
3 & -2 & 1 & 0 \\
-1 & 1 & -1 & 1
\end{array}\right]
$$

и

$$
Q=\left[\begin{array}{cccc}
d_{1} & -3 d_{1} & 3 d_{1} & -d_{1} \\
-3 d_{1} & 9 d_{1}+d_{2} & -9 d_{1}-2 d_{2} & 3 d_{1}+d_{2} \\
3 d_{1} & -9 d_{1}-2 d_{2} & 9 d_{1}+4 d_{2}+d_{3} & -3 d_{1}-2 d_{2}-d_{3} \\
-d_{1} & 3 d_{1}+d_{2} & -3 d_{1}-2 d_{2}-d_{3} & d_{1}+d_{2}+d_{3}+d_{4}
\end{array}\right]
$$

Условия симметрии для второго столбца

$$
-3 d_{1}=3 d_{1}+d_{2}, \quad 9 d_{1}+d_{2}=-9 d_{1}-2 d_{2}
$$

сводятся к одному равенству

$$
d_{2}=-6 d_{1} .
$$

С учетом (11) косая симметрия третьего столбца требует выполнения соотношений

$$
3 d_{1}=-9 d_{1}+d_{3}, \quad 3 d_{1}=15 d_{1}-d_{3},
$$

означающих, что

$$
d_{3}=12 d_{1}
$$

Вследствие (11) и (12) обе средние компоненты четвертого столбца равны $-3 d_{1}$. Для его полной симметрии достаточно, чтобы

$$
d_{1}+d_{2}+d_{3}+d_{4}=-d_{1},
$$

т.е. чтобы

$$
d_{4}=-8 d_{1} .
$$

Итак, с точностью до множителя $d_{1}$

$$
Q=\left[\begin{array}{cccc}
1 & -3 & 3 & -1 \\
-3 & 3 & 3 & -3 \\
3 & 3 & -3 & -3 \\
-1 & -3 & -3 & -1
\end{array}\right]
$$


5. То обстоятельство, что в проведенных выкладках различные соотношения для очередного элемента $d_{i}$ всякий раз оказывались непротиворечивыми, можно объяснить с помощью тождеств, которьм подчиняются биномиальные коэффициенты. Покажем это, анализируя первые шаги аналогичного процесса при произвольном $m$, считая, для определенности, что $m=2 k+1$ - нечетное число.

Будем рассматривать матрицы $P$ и $Q$ как наборы из $m$ столбцов:

$$
P=\left[p_{1} \ldots p_{m}\right], \quad Q=\left[q_{1} \ldots q_{m}\right]
$$

Поскольку число $m$ нечетно, вектор $p_{1}$ симметричен (см. (5)). Согласно (3) имеем

$$
q_{2}=\alpha p_{1}+\beta p_{2}
$$

где (см. (4))

$$
\alpha=-(m-1) d_{1}, \quad \beta=d_{2} .
$$

Определим инверсию $m$-мерного вектора $x$ соотношением

$$
\widehat{x}=\mathscr{P}_{m} x \text {. }
$$

Применяя инверсию к обеим частям равенства (14) и учитьвая симметрию вектора $p_{1}$, получаем

$$
\widehat{q}_{2}=\alpha p_{1}+\beta \widehat{p}_{2} .
$$

Чтобы $q_{2}$ был кососимметричным вектором, должно вьполняться условие

$$
\beta\left(p_{2}+\widehat{p}_{2}\right)=-2 \alpha p_{1}
$$

Сравнивая первые компоненты и используя (15), находим

$$
d_{2}=-2(m-1) d_{1}
$$

Если $i \geqslant 2$, то $i$-я компонента левого вектора в (16) равна числу

$$
d_{2}(-1)^{i}\left(\left(\begin{array}{c}
m-2 \\
i-2
\end{array}\right)+\left(\begin{array}{c}
m-2 \\
m-i-1
\end{array}\right)\right)=d_{2}(-1)^{i}\left(\begin{array}{c}
m-1 \\
i-1
\end{array}\right)=-2 \alpha(-1)^{i-1}\left(\begin{array}{c}
m-1 \\
i-1
\end{array}\right)
$$

т.е. $i$-й компоненте вектора $-2 \alpha p_{1}$. Таким образом, справедливость равенства (16) обеспечивается соотношением (17). Его частными случаями являются равенства (8), (9) и (11).

Снова привлекая (3), имеем

$$
q_{3}=\gamma p_{1}+\delta p_{2}+\varepsilon p_{3}
$$

где

$$
\gamma=\frac{(m-1)(m-2)}{2} d_{1}, \quad \delta=-(m-2) d_{2}, \quad \varepsilon=d_{3} .
$$

Производя в (18) инверсию, получаем

$$
\widehat{q}_{3}=\gamma p_{1}+\delta \widehat{p}_{2}+\varepsilon \widehat{p}_{3} .
$$


Вектор $q_{3}$ будет симметричен, если

$$
\delta p_{2}+\varepsilon p_{3}=\delta \widehat{p}_{2}+\varepsilon \widehat{p}_{3}
$$

или

$$
\delta\left(p_{2}-\widehat{p}_{2}\right)=-\varepsilon\left(p_{3}-\widehat{p}_{3}\right) .
$$

Сравнение первых компонент приводит к равенству $\delta=\varepsilon$, т.е.

$$
d_{3}=-(m-2) d_{2}=2(m-1)(m-2) d_{1}=4\left(\begin{array}{c}
m-1 \\
2
\end{array}\right) d_{1} .
$$

Заметим, что это согласуется с (10) и (12).

Вторые компоненты векторов $p_{2}-\widehat{p}_{2}$ и $p_{3}-\widehat{p}_{3}$ равны соответственно $3-m$ и $m-3$, т.е. условие (20) вьполняется при $\delta=\varepsilon$ и для вторых компонент. Наконец, при $i \geqslant 3$ находим

$$
\begin{aligned}
\left\{p_{2}-\widehat{p}_{2}\right\}_{i} & =(-1)^{i}\left(\left(\begin{array}{c}
m-2 \\
i-2
\end{array}\right)-\left(\begin{array}{c}
m-2 \\
m-i-1
\end{array}\right)\right) \\
& =(2 i-m-1) \frac{(m-2) !}{(i-1) !(m-i) !}=-\left\{p_{3}-\widehat{p}_{3}\right\}_{i}
\end{aligned}
$$

Итак, при $\delta=\varepsilon$ векторное равенство (20) справедливо.

Аналогичным образом обосновывается возможность выбора последующих элементов $d_{i}, i \geqslant 4$. Однако тождества для биномиальных коэффициентов, позволяющие такой выбор, с ростом $i$ постепенно усложняются. Так, чтобы найти $d_{4}$, нужно показать, что при некоторых коэффициентах $\lambda, \mu, \nu, \pi$ имеет место векторное равенство

$$
2 \lambda p_{1}+\mu\left(p_{2}+\widehat{p}_{2}\right)+\nu\left(p_{3}+\widehat{p}_{3}\right)+\pi\left(p_{4}+\widehat{p}_{4}\right)=0 .
$$

Здесь

$$
\lambda=-\left(\begin{array}{c}
m-1 \\
3
\end{array}\right) d_{1}, \quad \mu=\left(\begin{array}{c}
m-2 \\
2
\end{array}\right) d_{2}, \quad \nu=-\left(\begin{array}{c}
m-3 \\
1
\end{array}\right) d_{3}, \quad \pi=d_{4} .
$$

Само значение $d_{4}$ проще всего определить из сравнения первых компонент в $(22)$ с использованием соотношений (17) и (21). Это дает

$$
d_{4}=-8\left(\begin{array}{c}
m-1 \\
3
\end{array}\right) d_{1}
$$

что согласуется (при $m=4)$ с (13).

\section{СПИСОК ЦИТИРОВАННОЙ ЛИТЕРАТУРЫ}

[1] Taussky O., Todd J. Another look at a matrix of Mark Kac // Linear Algebra Appl. 1991. V. 150. P. 341-360.

[2] Икрамов Х. Д. О матрицах, спектр которых предсказуемым образом реагирует на перестановки столбцов // Докл. РАН. 1999. Т. 364. № 3. С. 310-312.

[3] Taussky O., Zassenhaus H. On the similarity transformation between a matrix and its transpose // Pacific J. Math. 1959. V. 9. №3. Р. 893-896.

[4] Икрамов Х.Д. О конечных спектральных процедурах в линейной алгебре // Программирование. 1994. №1. С. 56-69. 